\title{
Swift Monitoring of M51: A 38 day Superorbital Period for the Pulsar ULX7 and a New Transient Ultraluminous X-Ray Source
}

\author{
Murray Brightman ${ }^{1}$, Hannah Earnshaw ${ }^{1}$ (D), Felix Fürst ${ }^{2}$ (D), Fiona A. Harrison ${ }^{1}$, Marianne Heida $^{3}$ (D), Gianluca Israel ${ }^{4}$ (D), \\ Sean Pike ${ }^{1}$ (1), Daniel Stern ${ }^{5}$ (iD, and Dominic J. Walton ${ }^{6}$ (1) \\ ${ }^{1}$ Cahill Center for Astrophysics, California Institute of Technology, 1216 East California Boulevard, Pasadena, CA 91125, USA \\ ${ }^{2}$ European Space Astronomy Centre (ESA/ESAC), Operations Department, Villanueva de la Cañada (Madrid), Spain \\ ${ }^{3}$ European Southern Observatory, Karl-Schwarzschild-Straße 2, D-85748 Garching bei München, Germany \\ ${ }^{4}$ INAF-Osservatorio Astronomico di Roma, via Frascati 33, I-00078 Monteporzio Catone, Italy \\ 5 Jet Propulsion Laboratory, California Institute of Technology, Pasadena, CA 91109, USA \\ ${ }^{6}$ Institute of Astronomy, Madingley Road, Cambridge CB3 OHA, UK \\ Received 2019 December 9; revised 2020 February 19; accepted 2020 March 4; published 2020 June 4
}

\begin{abstract}
We present the results from a monitoring campaign made with the Neil Gehrels Swift Observatory of the M51 galaxies, which contain several variable ultraluminous X-ray sources (ULXs). The ongoing campaign started in 2018 May, and we report here on $\sim 1.5 \mathrm{yr}$ of observations. The campaign, which consists of 106 observations, has a typical cadence of 3-6 days, and has the goal of determining the long-term X-ray variability of the ULXs. Two of the most variable sources were ULX7 and ULX8, both of which are known to be powered by neutron stars that are exceeding their isotropic Eddington luminosities by factors of up to 100. This is further evidence that neutron-starpowered ULXs are the most variable. Our two main results are, first, that ULX7 exhibits a periodic flux modulation with a period of 38 days varying over a magnitude and a half in flux from peak to trough. Since the orbital period of the system is known to be 2 days, the modulation is superorbital, which is a near-ubiquitous property of ULX pulsars. Second, we identify a new transient ULX, M51 XT-1, the onset of which occurred during our campaign, reaching a peak luminosity of $\sim 10^{40} \mathrm{erg} \mathrm{s}^{-1}$, before gradually fading over the next $\sim 200$ days until it slipped below the detection limit of our observations. Combined with the high-quality Swift/X-ray Telescope lightcurve of the transient, serendipitous observations made with Chandra and XMM-Newton provide insights into the onset and evolution of a likely super-Eddington event.
\end{abstract}

Unified Astronomy Thesaurus concepts: X-ray point sources (1270); X-ray transient sources (1852); X-ray photometry (1820); X-ray bright point (1812); X-ray active galactic nuclei (2035); Transient sources (1851); Light curves (918); Galaxy nuclei (609); Active galactic nuclei (16)

\section{Introduction}

The galaxies of M51, which consist of the main spiral galaxy M51a/NGC 5194 and its smaller companion M51b/ NGC 5195, are a host to a multitude of X-ray sources (e.g., Brightman et al. 2018a). These include two active galactic nuclei (AGNs) powered by accreting supermassive black holes (SMBHs), one at the center of each of the galaxies; several ultraluminous X-ray sources (ULXs); and transient sources, such as the type IIb supernova SN 2011dh.

Both SMBHs are weakly accreting, relatively speaking, with Eddington ratios $<10^{-4}$, and the AGN powered by the SMBH in M51a is heavily obscured by Compton thick material. The ULXs, in contrast, are believed to be strongly accreting compact objects. Indeed, two of them are known to be powered by neutron stars (NSs) far less massive than the SMBHs but apparently equally as powerful. For ULX8, the NS identification was determined from the detection of a cyclotron resonance scattering feature (CRSF), caused by interactions of charged particles with a strong magnetic field (Brightman et al. 2018b). For ULX7, this was determined from the detection of coherent pulsations from the source (Rodríguez Castillo et al. 2019).

The observational properties of ULXs originally suggested they were good intermediate-mass black hole candidates (e.g., Earnshaw et al. 2016). However, the detection of coherent pulsations and CRSFs mean that some of them are certainly not black holes, since black holes are unable to produce such signals. ULXs powered by NSs are a surprising phenomenon since their luminosities imply extreme Eddington ratios of up to 500, and are not a well-understood phenomenon.

The first NS ULX to be identified was M82 X-2 (Bachetti et al. 2014), followed by NGC 7793 P13, NGC 5907 ULX1 and NGC 300 ULX1 (Fürst et al. 2016; Israel et al. 2017a, 2017b; Carpano et al. 2018), with the most recent discovery being NGC 1313 X-2 (Sathyaprakash et al. 2019). Thus M51 ULX7 and ULX8 are among a small but growing group of these sources. Being hosted in the same galaxy and separated by a few arcminutes means they can be easily studied together.

No pulsations have been detected from ULX8 yet, and hence a spin period is unknown. The spin period of ULX7 is $\sim 2 \mathrm{~s}$, which is similar to the $\sim 1 \mathrm{~s}$ spin periods for M82 X-2, NGC 7793 P13 and NGC 5901 ULX1. These sources also show another distinguishing characteristic: $\mathrm{X}$-ray flux modulations with periods of 60-80 days. These were all detected from long-term monitoring by the Neil Gehrels Swift Observatory (hereafter Swift; Qiu et al. 2015; Walton et al. 2016b; Hu et al. 2017; Fürst et al. 2018). Theories to explain these invoke a precession of the accretion disk or wind and some geometric beaming (e.g., Dauser et al. 2017; Middleton et al. 2018).

Furthermore, NGC 7793 P13 and NGC 5901 ULX1 exhibit unusual "off" states in addition to the periodic modulations, where the X-ray flux drops significantly, by orders of magnitude, relative to their peak brightness (Motch et al. 
2014; Walton et al. 2015). While the nature of these off states is currently unknown, Tsygankov et al. (2016) suggest they are related to the onset of the propeller effect, in which the magnetospheric radius moves outside the corotation radius such that accretion is dramatically suppressed and the X-ray flux drops precipitously.

Since the discovery that M51 harbors two ULXs powered by NSs, we have obtained monitoring of the galaxies with Swift in order to identify potential periodic flux modulations and off states exhibited by the other sources of this class. We present the results of this monitoring campaign here. In addition to ULX7 and ULX8, ULX4 was identified as a source of interest since it exhibits a bimodal distribution of fluxes, possibly related to the propeller effect (Earnshaw et al. 2018), and Urquhart \& Soria (2016) identified two eclipsing ULXs in the galaxy. Finally, M51 has also hosted several transient phenomena such as the type II supernova SN 2005cs, the type IIb supernova SN 2011dh and the intermediate luminosity red transient AT2019abn (Jencson et al. 2019). The Swift monitoring also allows us to study these other interesting ULXs and to look for transients in the X-ray band. We assume a distance of $8.58 \mathrm{Mpc}$ to M51 throughout this study (McQuinn et al. 2016). During the preparation of this manuscript, Vasilopoulos et al. (2020) was published presenting results similar to ours regarding ULX7 based on the publicly available Swift data. Our analyses differ, however, since they use the online tool described in Evans et al. (2009), to calculate the X-ray Telescope (XRT) count rates, whereas we use our own tailored analysis method. We also present $\sim 100$ days more data than they do, covering an extra three cycles of the modulation.

\section{Swift/XRT Data Analysis}

We have conducted a systematic monitoring campaign of the M51 galaxies with Swift/XRT (Burrows et al. 2005) since 2018 May 1 with a typical cadence of 3-6 days and typical exposure time of $2000 \mathrm{~s}$ per snapshot. Since our main goal was to investigate the long-term X-ray lightcurves of the two NSpowered ULXs, ULX7 and ULX8, to look for periodic flux modulations, the observing campaign was designed with this goal in mind. However, as described above, the M51 galaxies contain many other interesting X-ray sources which we have also obtained long-term lightcurves for.

In order to determine which additional sources are bright enough to provide useful lightcurves, we stack all 106 observations using the online XRT analysis tool. We create images in three bands, $0.3-1,1-2.5$, and $2.5-10 \mathrm{keV}$. The image is presented in Figure 1 where red, green, and blue represent these three bands, respectively. The AGN of the two galaxies, M51a and M51b, and several known ULXs are clearly detected, as well as SN 2011dh and a previously uncataloged ULX which we call M51 XT-1.

We proceed to extract spectra from all sources using the HEASOFT v6.25 tool XSELECT. Source events are selected from a circular region with a $25^{\prime \prime}$ radius and background regions consisting of large circles external to the galaxies are used to extract background events. All extraction regions are shown in Figure 2. For each source spectrum, we construct the auxiliary response file using xrtmkarf. The relevant response matrix file from the CALDB is used. All spectra are grouped with a minimum of 1 count per bin.

We use the HEASOFT tool XSPEC to calculate backgroundsubtracted count rates in the $0.3-10 \mathrm{keV}$ band. The lightcurves of these sources are shown in Figure 3. For observations where a source has zero total counts, we estimate the $90 \%$ upper limit on the count rate using a typical background count rate of $7 \times 10^{-5}$ counts $^{-1}$ and Poisson statistics.

Our next step is to look for count rate variability in the sources. We do this by testing for deviations from a constant count rate by calculating the $\chi^{2}$ of the count rates where

$$
\chi^{2} \equiv \sum_{n=1}^{N_{\mathrm{obs}}}\left(\frac{\mathrm{CR}_{n}-\langle\mathrm{CR}\rangle}{\sigma_{n}}\right)^{2}
$$

$\mathrm{CR}_{n}$ is the count rate in each observation, $n,\langle\mathrm{CR}\rangle$ is the mean count rate averaged over all observations, and $\sigma_{n}$ is the $1 \sigma$ uncertainty on the count rate for each observation. We present the results in Table 1. We find that the sources which exhibit evidence for count rate variability, which we arbitrarily define as $\chi_{r}^{2} \equiv \chi^{2} / N_{\text {obs }}>2.0$, are ULX4, ULX7, ULX8, the eclipsing ULXs, and M51 XT-1. The sources with the largest variability (i.e., $\chi_{r}^{2}>10.0$ ) are indeed ULX7 and ULX8.

Following this, we look for periodic flux modulations from all the sources with evidence for variability. We use two techniques to do so. First, we do epoch folding, whereby the lightcurve is folded on several test periods and deviations from a constant flux are determined (Leahy 1987). This technique is well suited for finding periodic signals which are not necessarily sinusoidal. We also use a Lomb-Scargle periodogram which is commonly used to search for periodicities in unevenly sampled data and is specialized for finding sinusoidal signals (Lomb 1976; Scargle 1982).

For the epoch-folding analysis, we search for periods ranging from 20-100 days over 200 linearly spaced steps. For each test period we split the lightcurve into 10 phase bins, as has been common practice in these searches and compute the $L$-statistic (Davies 1990). For the Lomb-Scargle analysis, we search over the same period range with the same number of test periods. As shown in Figure 4, ULX7 has a strong peak at 38 days in the periodogram seen from both epoch folding and the Lomb-Scargle analysis. A peak at twice this period is also seen in the epoch-folding periodogram, which is likely a harmonic of the shorter period peak, but we discuss the possibility that it is the fundamental later. There are no strong peaks seen in any other of the variable sources.

\subsection{Significance Simulations}

In order to determine the significance of the signals exhibited in the periodograms, we conduct simulations to determine the false alarm rate, i.e., the rate at which such a signal could be produced from a statistical fluctuation rather from a real signal. While both the epoch folding using the $L$-stat and the LombScargle periodogram define tests to assess the significance of signals, they can often overestimate the significance. Therefore, we follow Walton et al. (2016a) by simulating 10,000 lightcurves with $2000 \mathrm{~s}$ resolution and a red noise power spectrum and we sample them with the same observational strategy as the real lightcurves, running the same analysis as described above. We then note the largest peak in each periodogram, irrespective of period. We determine the $99.9 \%$ significance level by calculating at what level $99.9 \%$ of the simulated periodograms fall under. We plot this quantity on Figure 4, which shows that the 38 day period is detected with a significance greater than $99.9 \%$. 


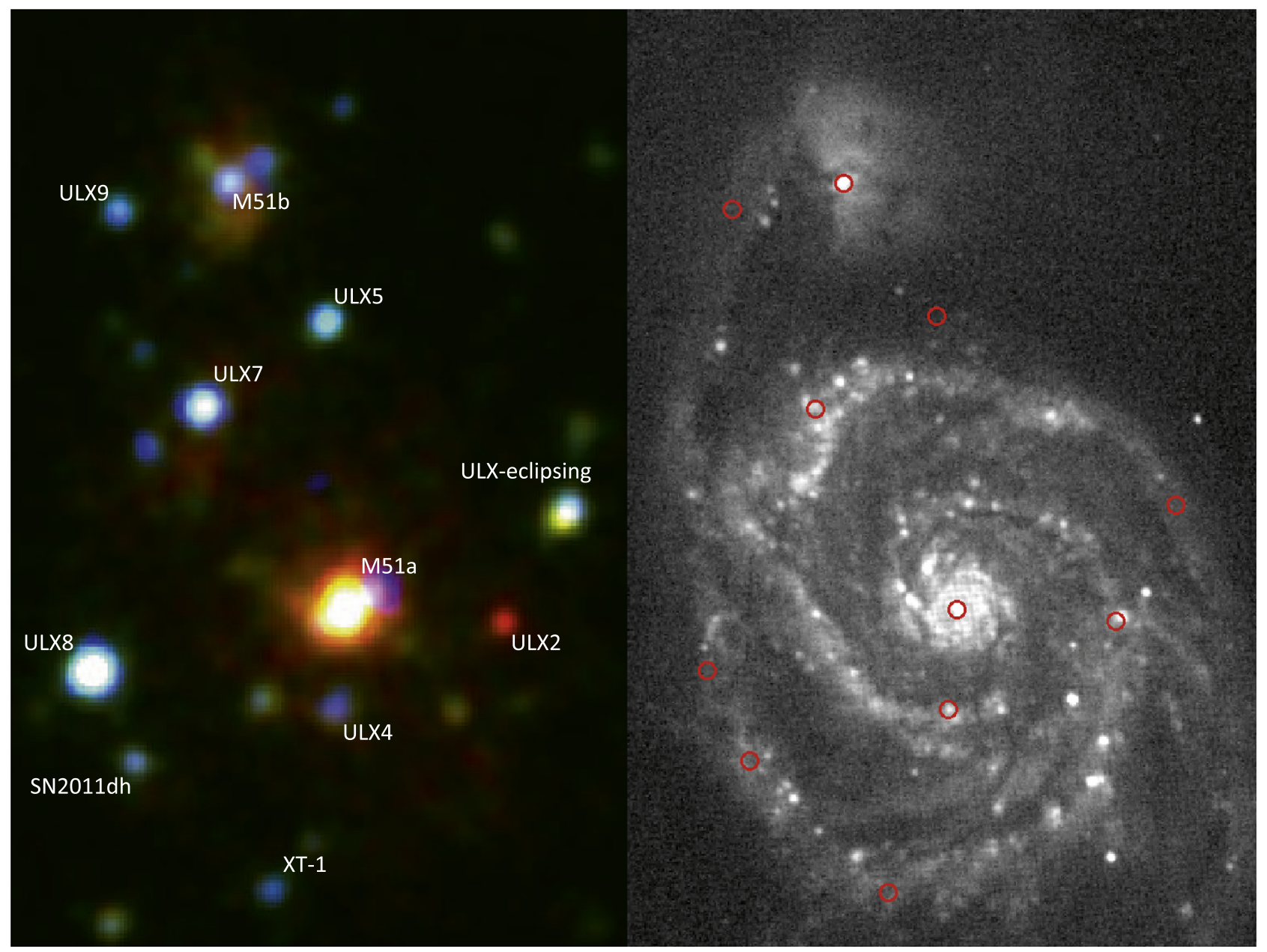

Figure 1. Left: stacked Swift/XRT images of the M51 galaxies. Red represents $0.3-1 \mathrm{keV}$ emission, green represents $1-2.5 \mathrm{keV}$ emission, and blue represents 2.5-10 keV emission. The image has been smoothed with a 4" Gaussian and the sources of study have been labeled. Right: Swift/Ultraviolet and Optical Telescope (UVOT) obsID $00032017112 u$-band image of M51 with the same sources marked with red circles.

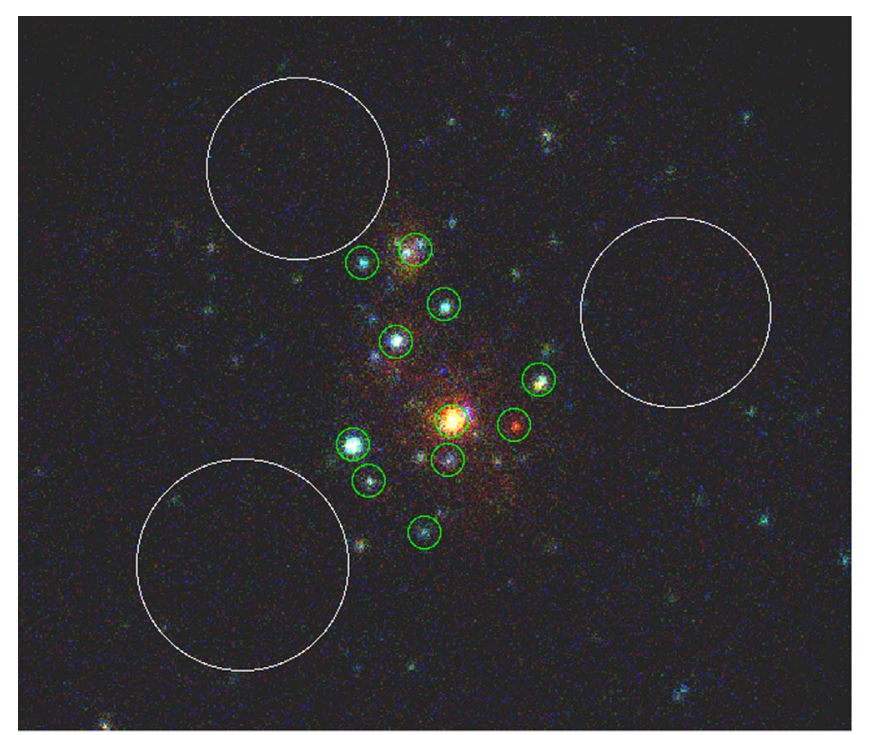

Figure 2. Same as Figure 1 (left), but image is wider angle, not smoothed, and shows the source (green) and background (white) extraction regions.

\section{Investigating the Periodic Flux Modulation from ULX7}

The orbital period of the NS powering ULX7 and its binary companion is known to be 2 days, which was determined from the timing analysis of the pulsations (Rodríguez Castillo et al. 2019). Therefore, the 38 day periodic flux modulation we detect here from ULX7 is superorbital in nature, making it the fourth ULX pulsar where this characteristic has been identified. Interestingly, ULX8 does not exhibit any periodic flux modulations on the timescales we have searched.

Investigating further, from the epoch-folding analysis described above, we determine the average profile of the modulation by calculating the mean count rate in each of the 10 phase bins. The average profile is plotted in Figure 5 and appears sinusoidal. The mean profile of the modulations peaks at $\sim 0.013$ counts s ${ }^{-1}$ and has a minimum at $\sim 0.001$ counts $\mathrm{s}^{-1}$. This is more than an order of magnitude from peak to trough, and corresponds to a luminosity range of $\sim 8 \times 10^{38}-10^{40}$ $\mathrm{erg} \mathrm{s}^{-1}$.

The deviations from this average profile, which we define as $\sigma=\frac{\text { data }- \text { profile }}{\text { error }}$, are also shown. These do not appear to show any long-term structure in addition to the sinusoidal modulation, with the exception of three dips in the lightcurve where $\sigma<-4$. Also plotted in Figure 5 are the average profile and data plotted against phase, as well as the deviation from the profile. The dip features are clustered at phase $\sim 0.8-1$, but further monitoring is required to determine if these features are coherent in phase of the flux modulation. 


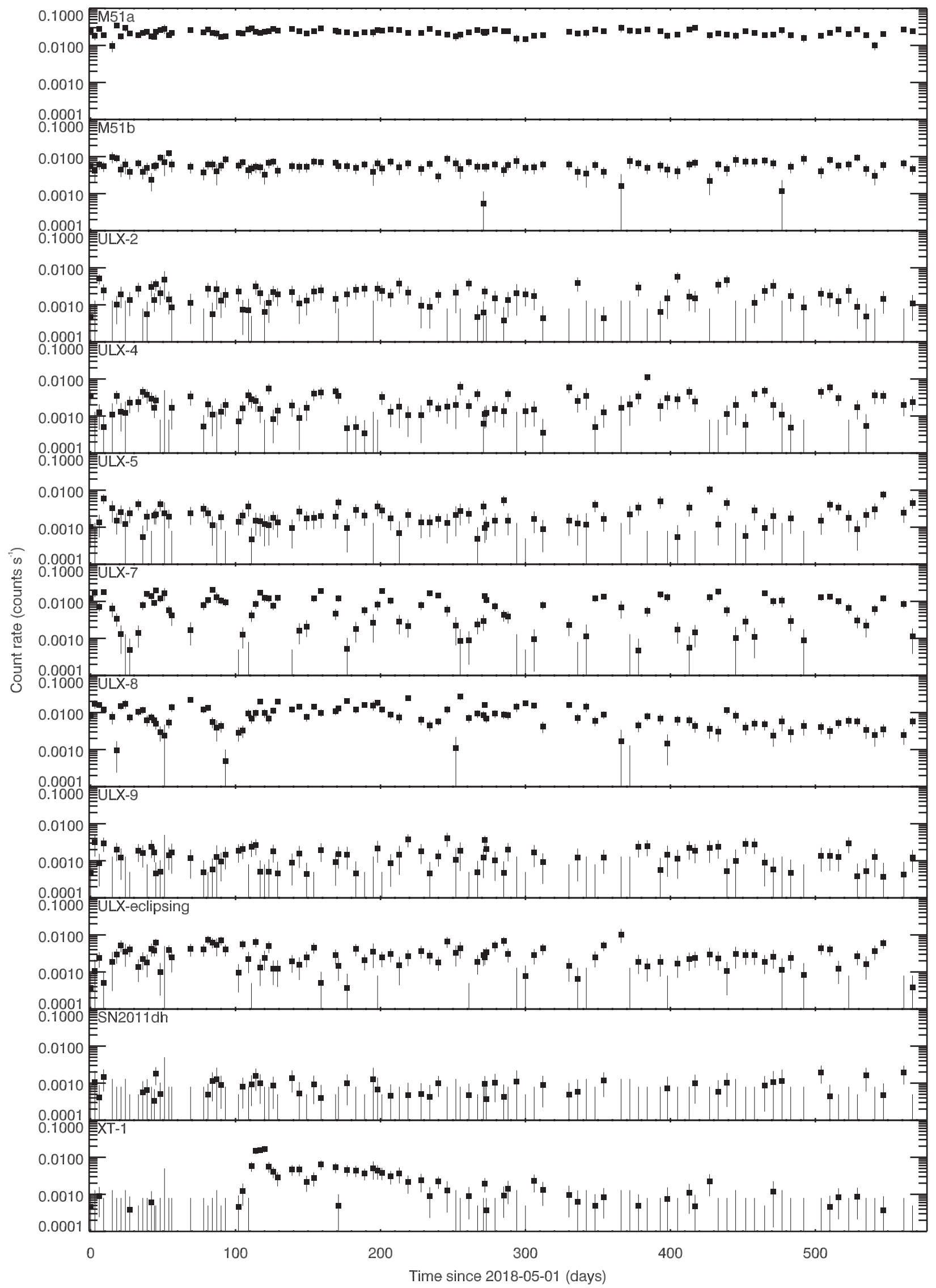

Figure 3. 0.3-10 keV Swift/XRT lightcurves of all sources of interest in M51 presented in counts per second as black squares with $1 \sigma$ error bars. $90 \%$ upper limits for nondetections are shown as error bars without squares. 
Table 1

Source Statistics

\begin{tabular}{|c|c|c|c|c|c|c|}
\hline $\begin{array}{l}\text { Source Name } \\
\text { (1) }\end{array}$ & $\begin{array}{l}\text { Position } \\
\text { (2) }\end{array}$ & $\begin{array}{l}\text { SIMBAD Identifier } \\
\text { (3) }\end{array}$ & $\begin{array}{c}\langle\mathrm{CR}\rangle \\
(4)\end{array}$ & $\begin{array}{l}\chi^{2} \\
(5)\end{array}$ & $\begin{array}{l}N_{\text {obs }} \\
\text { (6) }\end{array}$ & $\begin{array}{l}\chi_{r}^{2} \\
(7)\end{array}$ \\
\hline M51a & $13: 29: 52.773,+47: 11: 42.08$ & M51 & 0.0226 & 151.5 & 109 & 1.389 \\
\hline M51b & $13: 29: 59.221,+47: 15: 58.57$ & NGC 5195 & 0.0057 & 181.2 & 109 & 1.662 \\
\hline ULX-4 & $13: 29: 53.300,+47: 10: 42.00$ & [TW2004] NGC 519437 & 0.0021 & 271.0 & 109 & 2.486 \\
\hline ULX-5 & $13: 29: 53.937,+47: 14: 38.40$ & RX J132954+47145 & 0.0020 & 207.7 & 109 & 1.906 \\
\hline ULX-7 & $13: 30: 01.098,+47: 13: 42.33$ & RX J133001+47137 & 0.0071 & 2832.2 & 109 & 25.983 \\
\hline ULX-eclipsing & $13: 29: 39.861,+47: 12: 44.75$ & RX J132939+47126 & 0.0027 & 382.8 & 109 & 3.512 \\
\hline SN 2011dh & $13: 30: 04.993,+47: 10: 11.21$ & SN 2011dh & 0.0004 & 46.7 & 109 & 0.428 \\
\hline XT-1 & $13: 29: 56.843,+47: 08: 52.60$ & $\ldots$ & 0.0015 & 391.0 & 109 & 3.587 \\
\hline
\end{tabular}

Note. Summary of the sources studied here. Column (1) lists the source name adopted, column (2) gives the position in J2000 coordinates, column (3) lists the SIMBAD identifier, column (4) gives the mean 0.3-10 keV Swift/XRT count rate in counts per second, column (5) shows the $\chi^{2}$ of the count rate, column (6) gives the number of observations used, and column (7) gives the reduced $\chi^{2}$.

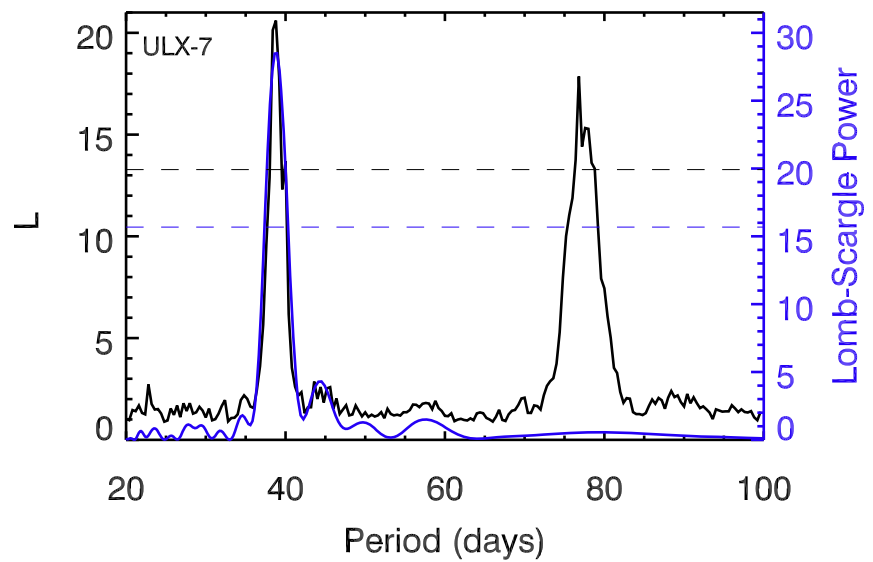

Figure 4. Results from the epoch-folding (black) and Lomb-Scargle analysis (blue) on the lightcurve of ULX7. A clear signal at 38 days is seen in both periodograms of this source, which is significant at $>99 \%$ significance, based on simulations (dashed lines).

We next look for spectral variations as a function of the phase of the flux modulation, which may yield insight into their origin. While the individual $\sim 2 \mathrm{ks}$ Swift/XRT observations do not have the photon statistics to conduct spectral analysis, we calculate hardness ratios for each, where $\mathrm{HR}=(H-S)$ / $(H+S), S$ is the total number of counts in the $0.3-2 \mathrm{keV}$ band and $H$ is the total number of counts in the $2-10 \mathrm{keV}$ band. We show the results in Figure 6, where we also display the binned averages. We find that the source is relatively hard during the peaks of the modulation, and relatively soft during the troughs. The individual point with $\mathrm{HR}>0.3$ is likely an outlier. The Swift/XRT exposure is only $809 \mathrm{~s}$, and when the spectrum is fitted with a power law, the power-law index is essentially unconstrained between 0.1 and 2.3.

\section{A New Transient ULX}

In addition to the well-known bright X-ray sources in M51, we detect a new, previously uncatalogued source when stacking the XRT images (Figure 1). The new source appeared in the southern part of M51a at coordinates $13^{\mathrm{h}} 29^{\mathrm{m}} 56^{\mathrm{s}} .8,+47^{\circ}$ $08^{\prime} 52$ ". 6 (J2000), and we refer to it as M51 XT-1. The lightcurve of the source in Figure 3 shows that it was a transient. The source was weakly detected, or undetected during the first $\sim 100$ days of the monitoring campaign, with a count rate $<0.001$ counts $\mathrm{s}^{-1}$, but became one of the brightest $\mathrm{X}$-ray sources in the galaxy with a count rate of $\sim 0.02$ counts $s^{-1}$ in the space of 10-15 days. The peak of its activity occurred during a 6 day period covering 2018 August 23 to 29 . The source then gradually faded over the next $\sim 200$ days and was once again weakly detected at day $\sim 400$ of our monitoring campaign.

There are no known, cataloged sources at the position of M51 XT-1, with no sources within $20^{\prime \prime}$ in the Chandra source catalog of Kuntz et al. (2016), which is the deepest catalog of X-ray sources in M51 available, summing over $800 \mathrm{ks}$ of Chandra exposure time. There are also no matching sources in the latest ULX catalog of Earnshaw et al. (2019) compiled from the XMM-Newton serendipitous source catalog, nor in the XMM-Newton serendipitous source catalog itself (3XMM DR8).

We searched the Transient Name Server ${ }^{7}$ for known transients in M51, finding none that occurred in 2018. AT2019abn was the closest in time and spatial separation and was an intermediate luminosity red transient discovered on 2019 January 22 several arcminutes from M51 XT-1 (Jencson et al. 2019). Figure 1 shows the Swift/UVOT $u$-band image of M51 during the peak of the outburst: no source is seen at the position of M51 XT-1. We also looked at the UVOT data from later observations to check for delayed emission at longer wavelengths, but we did not see anything either.

Using the build Swift/XRT spectral products tool ${ }^{8}$ (Evans et al. 2009), we created a spectrum of M51 XT-1 when it was at the peak of its outburst. We grouped the spectrum with a minimum of 1 count per bin and fitted it with an absorbed power law using the Cash statistic for minimization. The bestfit $C$-statistic was $C=65.32$ with 59 degrees of freedom (DoF), indicating the power-law model describes the spectrum adequately. The spectrum is shown in Figure 8 which is very hard with $\Gamma=1.1_{-0.5}^{+0.7}$ and no evidence for absorption above the Galactic value. The source had a peak flux of $1.2 \times 10^{-12}$ erg cm $\mathrm{cm}^{-2} \mathrm{~s}^{-1}$, which corresponds to a luminosity of $1.1 \times 10^{40}$ $\operatorname{erg~s}^{-1}$ at $8.58 \mathrm{Mpc}$.

\footnotetext{
https://wis-tns.weizmann.ac.il

8 http://www.swift.ac.uk/user_objects/
} 


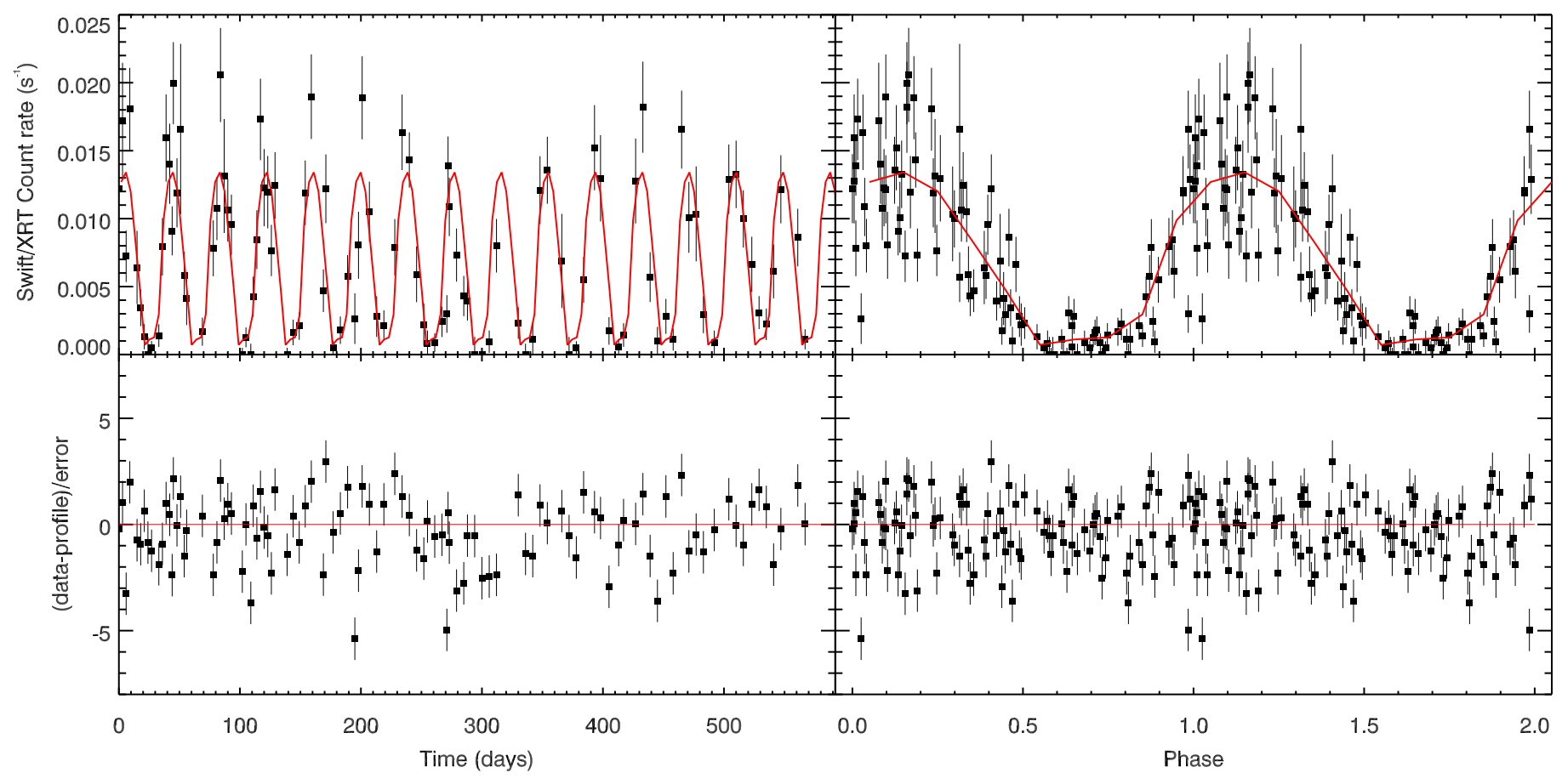

Figure 5. Top left: $0.3-10 \mathrm{keV}$ Swift/XRT lightcurve of ULX7 (black data points, $1 \sigma$ errors), overplotted with the average 38 day profile determined from epoch folding (red line). Top right: the same lightcurve but folded on the 38 day period and plotted against phase. Bottom left: deviations of the data from the average profile shown against time. Bottom right: deviations of the data from the average profile folded on the 38 day period.

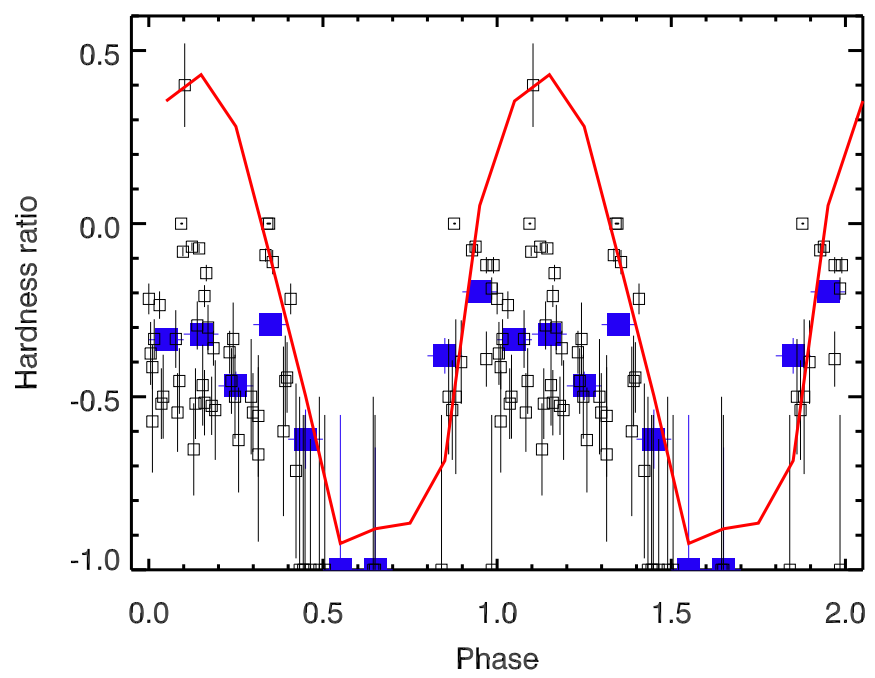

Figure 6. Hardness ratios of ULX7 as a function of phase of the superorbital period (black squares), with binned averages (blue open squares). The scaled average profile of the count rate is shown for reference (red line).

Serendipitously, Chandra observed M51 for 19.8 ks on 2018 August 31 (obsID 20998), only a few days after the peak of activity from M51 XT-1. This allowed us to obtain a better position of the source than Swift/XRT provided, and a higher signal-to-noise spectrum. We ran the CIAO tool wavdetect on the observation to obtain list of positions for all sources. We then cross-correlated this source list with the Gaia Data Release 2 catalog to obtain the astrometric shifts. Having applied the astrometric shifts to the Chandra source catalog, we determine the position of M51 XT-1 to be R.A. = 13:29:56.97, decl. $=+47: 08: 54.1(\mathrm{~J} 2000)$, with an astrometric uncertainty of 0 ." 45 from the residual offsets with the Gaia catalog.
We used the CIAO v4.11 tool SPECEXTRACT to extract the spectrum of the source from a circular region with a $2^{\prime \prime}$ radius. Background events were expected from a nearby region. The source had a count rate of $4.1 \pm 0.1 \times 10^{-2}$ counts s $^{-1}$ on the ACIS detector, and the spectrum is well-fitted by an absorbed power-law spectrum (tbabs*powerlaw) with $C=251.92$ and 292 DoFs, where $N_{\mathrm{H}}=3.3 \pm 0.1 \times 10^{21} \mathrm{~cm}^{-2}$ and $\Gamma=1.61 \pm 0.20$ with a flux of $7.2 \pm 0.7 \times 10^{-13}$ erg cm $\mathrm{cm}^{-2} \mathrm{~s}^{-1}$, which corresponds to a luminosity of $6.3 \times 10^{39} \mathrm{erg} \mathrm{s}^{-1}$ at $8.58 \mathrm{Mpc}$. The spectrum is shown in Figure 8 .

Additionally, XMM-Newton observed M51 on 2019 July 11 (obsID 0852030101), almost a year after the outburst, also serendipitously. The source was detected with a count rate of $4 \times 10^{-3}$ counts s$^{-1}$ in the pn detector. We extracted a spectrum of the source using a circular region with a radius of $10^{\prime \prime}$, and extracted the background from a nearby region, using the XMMSAS v18.0.0 tool EVSELECT. The source is described by a power-law spectrum with $N_{\mathrm{H}}=1.9_{-0.9}^{+1.1} \times 10^{21}$ $\mathrm{cm}^{-2}$ and $\Gamma=2.6 \pm 0.5$, where $C=187.49$ with $\mathrm{DoF}=254$, which presents a clear softening of the spectrum since the outburst. The source exhibited a flux of $2.0_{-0.5}^{+0.4} \times 10^{-14}$ erg cm$~^{-2} \mathrm{~s}^{-1}$, which corresponds to a luminosity of $2 \times 10^{38}$ $\mathrm{erg} \mathrm{s}^{-1}$ at $8.68 \mathrm{Mpc}$. The spectrum is also shown in Figure 8.

To place these spectra in context, we show in Figure 8 the spectrum of ULX7 which was obtained from a joint XMMNewton and NuSTAR observing campaign in 2019 (M. Brightman et al. 2020, in preparation). The spectrum of ULX7 is similar to that of other ULXs with good quality broadband spectra (e.g., Walton et al. 2018), consisting of two disk-like components with different temperatures. ULX7 also has a similar flux and luminosity as M51 XT-1 at its peak, therefore serving as a good comparison. As seen, the spectra of M51 XT-1 at its peak are harder than ULX7 and resemble the hot disk-like component seen in that source. Although the 


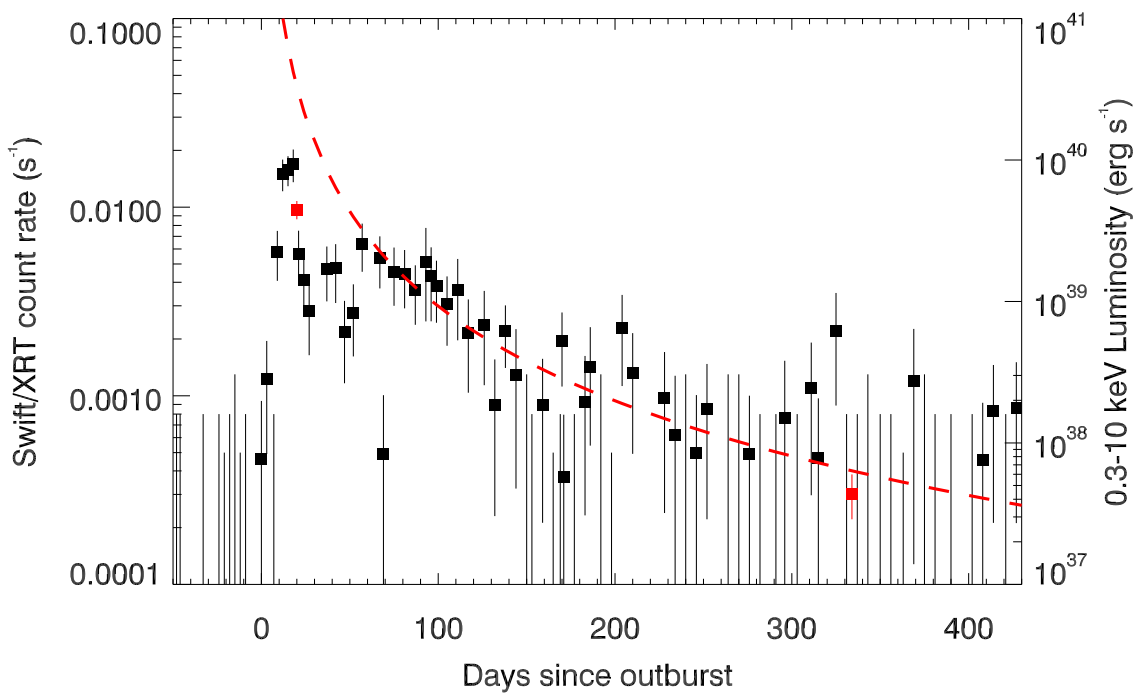

Figure 7. Lightcurve of M51 XT-1 during its outburst monitored by Swift/XRT (black squares), and serendipitously observed with Chandra and XMM-Newton (red squares). Also shown is a red dashed line with a $t^{-5 / 3}$ shape, which matches the data well after $\sim 50$ days of outburst.

Table 2

Superorbital Periods of All ULX Pulsars Known to Date

\begin{tabular}{lcccccccc}
\hline \hline $\begin{array}{l}\text { Source Name } \\
(1)\end{array}$ & $\begin{array}{c}P_{\text {spin }} \\
(2)\end{array}$ & $\begin{array}{c}\text { References } \\
(3)\end{array}$ & $\begin{array}{c}P_{\text {orbit }} \\
(4)\end{array}$ & $\begin{array}{c}\text { References } \\
(5)\end{array}$ & $\begin{array}{c}P_{\text {super-orbital }} \\
(6)\end{array}$ & $\begin{array}{c}\text { References } \\
(7)\end{array}$ & $\begin{array}{c}L_{\mathrm{X}}, \min -L_{\mathrm{X}}, \text { max } \\
(8)\end{array}$ & $\begin{array}{c}\text { References } \\
(9)\end{array}$ \\
\hline M82 X-2 & 1.37 & $(1),(2)$ & 2.5 & $(1),(2)$ & 64 & $(3)$ & $\sim 10^{38}-10^{40}$ \\
NGC 7793 P13 & 0.42 & $(5),(6)$ & 63.9 & $(7)$ & 66.8 & $(7)$ & $\sim 4 \times 10^{39}-10^{40}$ \\
NGC 5907 ULX1 & 1.43 & $(9)$ & 5.3 & $(9)$ & 78.1 & $(10)$ & $\sim 3 \times 10^{40}-10^{41}$ & $(10),(7)$ \\
M51 ULX7 & 2.8 & $(12)$ & 2.0 & $(12)$ & 38 & $(13)$ & $\sim 8 \times 10^{38}-10^{40}$ \\
\hline
\end{tabular}

Note. Summary of the observed properties of the ULX pulsars which exhibit superorbital periods in their lightcurves. Column (1) lists the source name, column (2) lists the spin period of the NS in seconds as determined from the pulsations, column (3) gives the reference for the spin, column (4) gives the orbital period in days of the NS and its binary companion determined from timing analyses of the pulsations, column (5) gives the reference for the orbital period, column (6) gives the superorbital period in days, column (7) gives the reference for this. Column (8) gives the luminosity range of the superorbital flux modulations in ergs per second and column (9) gives the reference.

References. (1) Bachetti et al. (2014), (2) Bachetti et al. (2020), (3) Brightman et al. (2019), (4) Brightman et al. (2016), (5) Fürst et al. (2016), (6) Israel et al. (2017b), (7) Fürst et al. (2018), (8) Walton et al. (2018), (9) Israel et al. (2017a), (10) Walton et al. (2016a), (11) Fürst et al. (2017), (12) Rodríguez Castillo et al. (2019), (13) This work.

absorption is higher for M51 XT-1 (3.3 compared to $0.7 \times 10^{21}$ $\mathrm{cm}^{-2}$ ), this does not account for all the spectral hardness as a cooler disk-like component can be ruled out in the Chandra spectrum. We discuss the physical implications of this further in Section 5.

Finally, XMM-Newton observed M51 four times (obsIDs 0824450901, 0830191401, 0830191501, and 0830191601) in a period of $\sim 100$ days before the outburst, allowing us to place tight upper limits on the flux of the source before outburst. For each individual observation, the upper limit on the $0.3-10 \mathrm{keV}$ flux is $\sim 8 \times 10^{-15} \mathrm{erg} \mathrm{cm}^{-2} \mathrm{~s}^{-1}$, corresponding to $L_{X}$ $\sim 7 \times 10^{37} \mathrm{erg} \mathrm{s}^{-1}$. Figure 7 shows the lightcurve of M51 XT-1 with the Chandra and XMM-Newton data added. We also show a line with a $t^{-5 / 3}$ shape that matches the data well after $\sim 50$ days of outburst for discussion in Section 5.

Using the astrometrically-corrected Chandra position of M51 XT-1, we searched the Hubble Source Catalog (HSCv3) for potential counterparts. Only one source is found within the 0 ". 45 positional uncertainty, at a position of 13:29:56.983, $+47: 08: 54.35$ detected by WFC3 in the F110W filter with a magnitude of 24.44. The distance modulus corresponding to $8.58 \mathrm{Mpc}$ is 29.7 , implying an absolute magnitude of this source of $M_{\mathrm{F} 110 \mathrm{~W}}=-5.22$.

\section{Discussion}

\subsection{A 38 day Superorbital Flux Modulation from ULX7}

The orbital period of the NS powering ULX7 and its binary companion is known to be 2 days, which was determined from the timing analysis of the pulsations (Rodríguez Castillo et al. 2019). Therefore, the 38 day periodic flux modulation we detect here from ULX7 is superorbital in nature, making it the fourth ULX pulsar where this characteristic has been identified. Indeed, it appears as if this characteristic is near-ubiquitous in these systems. This leads to the possibility that identifying periodic flux modulations in ULXs where the accretor is unknown can be used as strong evidence that it is powered by a NS. During the preparation of this manuscript, Vasilopoulos et al. (2020) also presented the identification of the 38 day superorbital period of M51 ULX7 from the same Swift data we have presented here.

We list the spin period, orbital period, and superorbital period for all ULX pulsars where these superorbital periods have been identified so far in Table 2 for reference and comparison. M51 ULX7 has the shortest superorbital period of the four, at 38 days, where the others are at 60-80 days. As mentioned earlier, the epoch-folding periodogram presents a peak at twice the period of the 38 day peak, which is most 
likely a harmonic, but if it is the true period, it would make it comparable to the other ULX pulsars. ULX7 also has the longest spin period for ULX pulsars with superorbital periods, at $2.8 \mathrm{~s}$, whereas the others have $0.4-1.4 \mathrm{~s}$. A larger sample is needed before any conclusions can be drawn regarding any inverse relationship between the spin period of the NS and the superorbital period.

The luminosity range of the flux modulation from ULX7, which is $\sim 8 \times 10^{38}-10^{40} \mathrm{erg} \mathrm{s}^{-1}$, is larger than NGC 7793 P13 and NGC 5901 ULX1 but smaller to M82 X-2. However, due to the observational challenges related to M82 X-2, ULX7 is much easier to study, making it the best source to study these extreme flux variations.

Not only does it appear that these superorbital periods are a near-ubiquitous property of ULX pulsars, but they have been observed in many other NS X-ray binary systems, such as Her X-1 (Tananbaum et al. 1972; Katz 1973), LMC X-4 (Lang et al. 1981), and SMC X-1 (Gruber \& Rothschild 1984) as well as black hole binaries (e.g., compilation by Sood et al. 2007). In these lower luminosity and lower mass-accretion rate systems, a warped precessing disk is often invoked to explain the flux modulations. Here the limb of the warped disk periodically occults the X-ray emitting region, causing the periodic dips in flux.

From our hardness ratio analysis, we found that the source is hard during the peaks of the modulation, and soft during the troughs. This argues against changes in the absorption causing the flux modulation, as this would have predicted the source being hard during the troughs, rather than soft, since absorption causes attenuation of soft X-rays. Occultation of the source by a warped disk would appear to be disfavored, unless the source is completely obscured during the occultations and we only see scattered emission.

Furthermore, it is likely that the thin accretion disk that is needed for warps does not exist in ULX pulsars. In these systems, the extreme accretion rate causes the disk to extend vertically giving it a large-scale height. Indeed, the precession of a large-scale height disk has been proposed as the mechanism behind the flux modulations in ULX pulsars (e.g., Dauser et al. 2017; Fürst et al. 2017; Middleton et al. 2018). This theory would also fit the observations of M51 ULX7 better. Here, where the source is at low fluxes and soft, we are potentially viewing the disk more edge on where only the cooler outer regions can be seen. When the source is at high fluxes, we see into the hot funnel region. This would require a very large precession angle, however, to go from a disk seen almost face on, to edge on.

Middleton et al. (2018) suggest that the precession can be explained by the Lense-Thirring effect, a consequence of general relativity whereby a massive spinning object induces a precession of orbiting particles that are displaced vertically from the rotation axis, for example, a large-scale height accretion flow. Indeed, Middleton et al. (2019) suggest that these timescale periodicities can be used to determine whether the compact object is a black hole or a NS.

Four XMM-Newton observations of M51 took place during the period 2018 May 13 to June 15, which covered the first cycle of the flux modulation we observed with Swift. Indeed, it was from these observations that the pulsations confirming ULX7 to be powered by a NS were detected (Rodríguez Castillo et al. 2019). Rodríguez Castillo et al. (2019) also presented a spectral analysis of the source from these data, finding too that the source became very soft during the minimum of the flux modulation. They also suggest that the luminosity swing may be caused by a change in inclination angle of the disk, though the scaling of the temperature of the disk with luminosity did not fit this hypothesis. Alternatively they proposed that the periodic flux modulations may be induced by changes in accretion rate caused by the orbit of a third star, although this explanation did not account for the spectral variations.

Interestingly, Earnshaw et al. (2016) presented the results from a detailed systematic spectral analysis of ULX7 using XMM-Newton, Chandra, and NuSTAR, finding no evidence for significant spectral evolution, even at the lowest fluxes. They did however find evidence for soft diffuse thermal emission surrounding the source in the Chandra data, that is likely contaminating the larger PSFs of XMM-Newton and Swift. It is possible that this component dominates the XMMNewton and Swift data at lower fluxes, making the source appear soft.

\subsection{The Nature of the New Transient ULX M51 XT-1}

The peak luminosity and timescale of M51 XT-1, the new transient we have identified, corresponds to the "ULX in outburst" section of the X-ray transients luminosity-timescale plot from Soderberg et al. (2009). However, we note that the decline of the outburst after $\sim 50$ days is consistent with a $t^{-5 / 3}$ relationship, which is the signature of the fallback from a tidal disruption event (TDE; Figure 7). TDEs are however much brighter and occur in the nuclei of galaxies, thought to be due to the tidal disruption of a star by a SMBH.

Other examples of transient ULXs are NGC 300 ULX1, which had an X-ray outburst in 2010, reaching an X-ray luminosity of $5 \times 10^{38} \mathrm{erg} \mathrm{s}^{-1}$ (Binder et al. 2011). The source was observed at lower fluxes in observations made in 2014 (Binder et al. 2016) but then reached ULX luminosities during observations made in 2016 with $L_{X} \sim 5 \times 10^{39}$ $\mathrm{erg} \mathrm{s}^{-1}$, during which time pulsations were detected (Carpano et al. 2018). Regular Swift monitoring of the source in 2018 revealed that the source initially persisted at these luminosities but then went into decline. Spectral analysis showed a hard spectrum.

Another source, Swift J0243.6+6124, was an X-ray transient found in our own Galaxy, identified by Swift/Burst Alert Telescope (Cenko et al. 2017) and with no previously reported activity. The source reached an X-ray luminosity of $2 \times 10^{39}$ $\mathrm{erg} \mathrm{s}^{-1}$ in a period of around 30 days, before steadily declining over a period of $\sim 100$ days (Wilson-Hodge et al. 2018). The detection of pulsations identified it as an NS accretor (Kennea et al. 2017). The source exhibited rebrightenings in the X-ray after the decline, albeit to peak luminosities around two orders of magnitude less than the initial outburst (van den Eijnden et al. 2019).

Most recently, Earnshaw et al. (2019) identified a new ULX in the galaxy NGC 6946 that was previously undetected in several archival XMM-Newton and Chandra observations, but caught at a luminosity of $2 \times 10^{39} \mathrm{erg} \mathrm{s}^{-1}$ during a simultaneous XMM-Newton and NuSTAR observation in 2017. The source was then undetected 10 days later in a Chandra observation. Again, the spectrum was hard with $\Gamma \sim 1$. Several hypotheses were put forward by Earnshaw et al. (2019) regarding the nature of this transient event, which may also explain M51 XT-1. These included a supernova, an NS- 


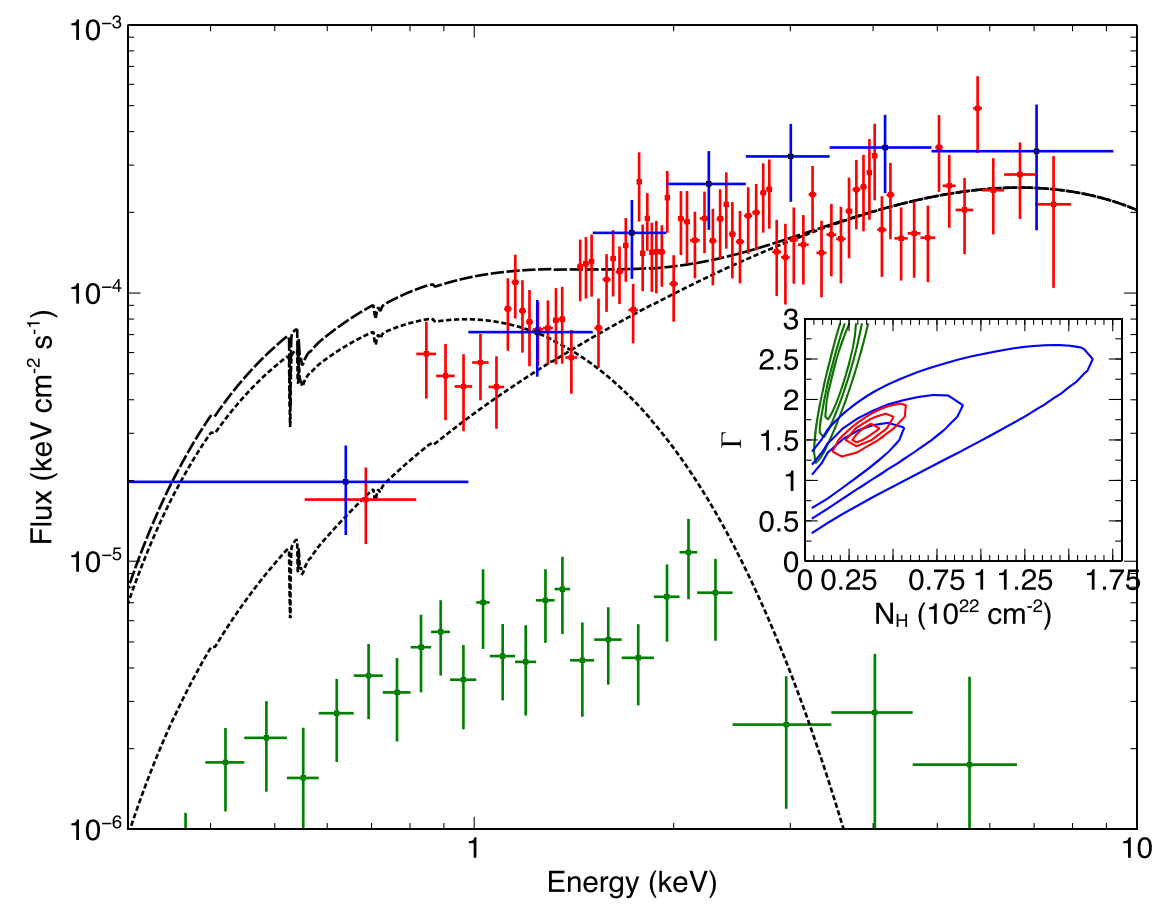

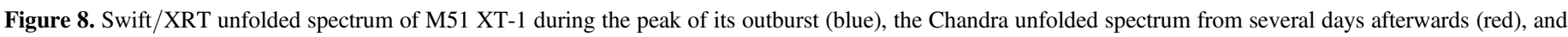

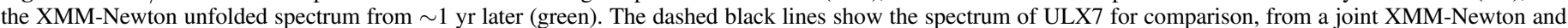

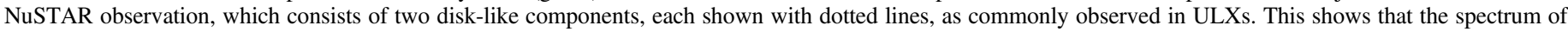

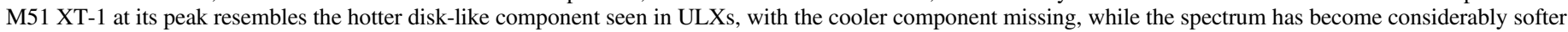
at late times. Inset are confidence contours resulting from a fit with an absorbed power law to the Swift, Chandra, and XMM-Newton spectra of M51 XT-1.

powered ULX briefly leaving the propeller regime, a black hole binary exhibiting a hard-only outburst, and a microtidal disruption event.

A supernova is unlikely to be the cause of M51 XT-1 since no supernova candidates were announced at its time and position and the galaxy is regularly monitored by transient surveys such as the Zwicky Transient Facility. Considering the peak luminosity of the outburst, a hard-only outburst would require a black hole much more massive than the black hole binaries in our Galaxy. The similarity of the outburst to other transient NS ULXs described above appears to favor that scenario, but the more exotic hypothesis of a microtidal disruption event cannot be ruled out, especially considering the $t^{-5 / 3}$ decline in count rate. It could be possible that the source is in the background of M51 and is in fact a nuclear TDE seen at larger distances, and hence luminosities. However, M51 XT1 is located in the spiral arm of M51, so the source should be absorbed, which is inconsistent with the X-ray spectrum.

Other examples of transient ULXs are ones in M31 (Middleton et al. 2012, 2013), M83 (Soria et al. 2012), NGC 1365 (Soria et al. 2007), NGC 3628 (Strickland et al. 2001), NGC 5907 (Pintore et al. 2018), and NGC 7090 (Liu et al. 2019), among others. For most of these transient ULXs, only a handful of observations were made and the lightcurves were not well sampled. Here, we serendipitously obtained a high-quality lightcurve that captured the onset of activity and covered it until it became undetected.

Since most ULXs are now considered to be super-Eddington accretors, we have likely witnessed the onset of a superEddington event with M51 XT-1 in M51, and the details we have gleaned from it may give clues regarding the formation of such an accretion flow. Most persistent ULXs show a characteristic broadband spectrum consisting of two disk-like components. Walton et al. (2018) presented a compilation of ULXs with good quality broadband X-ray spectra, finding that they are remarkably similar, even comparing those with known NS accretors and those with unknown accretors. The spectral components consist of two disk-like components, one potentially associated with the hot, large-scale-height inner flow, and the other a cooler component, perhaps associated with the outer part of the disk or the photosphere of an outflow. Furthermore, a high-energy tail is often detected, possibly associated with the accretion column of a pulsar, or Comptonized emission.

When comparing the spectral shape of M51 XT-1 during the peak of its activity to the ULX population, we noted that it resembled the hot disk-like component attributed to the largescale-height inner flow of other ULXs, with the cooler component absent (Figure 8). This perhaps suggests that the cooler component had yet to form. If the cooler component is indeed associated with the outer disk, its absence may explain why the outburst was relatively short, since it was not supplied by more material in the outer part of the disk. If instead the cooler component is from an outflow, it would imply that there is a time lag between the formation of the super-Eddington flow and the ejection of the material. Indeed a year after the outburst, the spectrum appeared much cooler, which may be the remnants of the outflow, albeit with the hotter component gone. Following an event such as this in future with more detail will allow a clearer picture of the formation and evolution of a super-Eddington flow. With the launch of eROSITA (Merloni et al. 2012), which will observe each part of the sky every 6 months, more of these transient ULXs will undoubtedly be identified. 


\section{Summary and Conclusions}

We have presented the results from the systematic monitoring of M51 by Swift/XRT over the period of $\sim 1.5 \mathrm{yr}$ which has yielded high-quality X-ray lightcurves of more than 10 $\mathrm{X}$-ray sources, including two AGN and several ULXs. Our main results are the detection of a 38 day superorbital flux modulation from the ULX pulsar, ULX7, and the identification of a new, transient ULX, M51 XT-1.

The superorbital period is a near-ubiquitous property of ULX pulsars, possibly driven by the precession of a large-scale height disk. However, the magnitude-and-a-half swings in flux are hard to reconcile with this theory. Alternatively, the spectral shape as a function of phase does not favor periodic obscuration of the source, unless it is being completely obscured and only scattered light is seen.

The outburst of M51 XT-1 appears similar to superEddington outbursts from other NSs, suggesting we have witnessed the onset and decline of a highly super-Eddington event. The spectral shape at the peak of the outburst is similar to the hot disk-like component seen in other ULXs that is attributed to a large-scale-height accretion flow.

We wish to thank the Swift PI, Brad Cenko for approving the target of opportunity requests we made to observe M51, as well as the rest of the Swift team for carrying them out. We also acknowledge the use of public data from the Swift data archive. This work made use of data supplied by the UK Swift Science Data Centre at the University of Leicester. The work of D.S. was carried out at the Jet Propulsion Laboratory, California Institute of Technology, under a contract with NASA. D.J.W. acknowledges support from an STFC Ernest Rutherford Fellowship.

\section{ORCID iDs}

Hannah Earnshaw (1) https://orcid.org/0000-0001-5857-5622

Felix Fürst (10 https://orcid.org/0000-0003-0388-0560

Marianne Heida (1) https://orcid.org/0000-0002-1082-7496

Gianluca Israel $\odot$ https://orcid.org/0000-0001-5480-6438

Sean Pike 주 https://orcid.org/0000-0002-8403-0041

Daniel Stern $\odot$ () https://orcid.org/0000-0003-2686-9241

Dominic J. Walton (1) https://orcid.org/0000-0001-5819-3552

\section{References}

Bachetti, M., Harrison, F. A., Walton, D. J., et al. 2014, Natur, 514, 202 Bachetti, M., Maccarone, T. J., Brightman, M., et al. 2020, ApJ, 891, 44 Binder, B., Williams, B. F., Kong, A. K. H., et al. 2011, ApJL, 739, L51 Binder, B., Williams, B. F., Kong, A. K. H., et al. 2016, MNRAS, 457, 1636 Brightman, M., Baloković, M., Koss, M., et al. 2018a, ApJ, 867, 110 Brightman, M., Harrison, F., Walton, D. J., et al. 2016, ApJ, 816, 60 Brightman, M., Harrison, F. A., Bachetti, M., et al. 2019, ApJ, 873, 115 Brightman, M., Harrison, F. A., Fürst, F., et al. 2018b, NatAs, 2, 312 Burrows, D. N., Hill, J. E., Nousek, J. A., et al. 2005, SSRv, 120, 165 Carpano, S., Haberl, F., Maitra, C., \& Vasilopoulos, G. 2018, MNRAS, 476, L45

Cenko, S. B., Barthelmy, S. D., D'Avanzo, P., et al. 2017, GCN, 21960, 1
Dauser, T., Middleton, M., \& Wilms, J. 2017, MNRAS, 466, 2236

Davies, S. R. 1990, MNRAS, 244, 93

Earnshaw, H. M., Roberts, T. P., Heil, L. M., et al. 2016, MNRAS, 456, 3840 Earnshaw, H. P., Roberts, T. P., Middleton, M. J., Walton, D. J., \& Mateos, S. 2019, MNRAS, 483, 5554

Earnshaw, H. P., Roberts, T. P., \& Sathyaprakash, R. 2018, MNRAS, 476, 4272

Evans, P. A., Beardmore, A. P., Page, K. L., et al. 2009, MNRAS, 397, 1177

Fürst, F., Walton, D. J., Harrison, F. A., et al. 2016, ApJL, 831, L14

Fürst, F., Walton, D. J., Heida, M., et al. 2018, A\&A, 616, A186

Fürst, F., Walton, D. J., Stern, D., et al. 2017, ApJ, 834, 77

Gruber, D. E., \& Rothschild, R. E. 1984, ApJ, 283, 546

Hu, C.-P., Li, K. L., Kong, A. K. H., Ng, C.-Y., \& Chun-Che Lin, L. 2017, ApJL, 835, L9

Israel, G. L., Belfiore, A., Stella, L., et al. 2017a, Sci, 355, 817

Israel, G. L., Papitto, A., Esposito, P., et al. 2017b, MNRAS, 466, L48

Jencson, J. E., Adams, S. M., Bond, H. E., et al. 2019, ApJ, 880, L20

Katz, J. I. 1973, NPhS, 246, 87

Kennea, J. A., Lien, A. Y., Krimm, H. A., Cenko, S. B., \& Siegel, M. H. 2017, ATel, 10809,

Kuntz, K. D., Long, K. S., \& Kilgard, R. E. 2016, ApJ, 827, 46

Lang, F. L., Levine, A. M., Bautz, M., et al. 1981, ApJL, 246, L21

Leahy, D. A. 1987, A\&A, 180, 275

Liu, Z., O’Brien, P. T., Osborne, J. P., Evans, P. A., \& Page, K. L. 2019, MNRAS, 486, 5709

Lomb, N. R. 1976, Ap\&SS, 39, 447

McQuinn, K. B. W., Skillman, E. D., Dolphin, A. E., Berg, D., \& Kennicutt, R. 2016, ApJ, 826, 21

Merloni, A., Predehl, P., Becker, W., et al. 2012, arXiv:1209.3114

Middleton, M. J., Fragile, P. C., Bachetti, M., et al. 2018, MNRAS, 475, 154

Middleton, M. J., Fragile, P. C., Ingram, A., \& Roberts, T. P. 2019, MNRAS, 489, 282

Middleton, M. J., Miller-Jones, J. C. A., Markoff, S., et al. 2013, Natur, 493, 187

Middleton, M. J., Sutton, A. D., Roberts, T. P., Jackson, F. E., \& Done, C. 2012, MNRAS, 420, 2969

Motch, C., Pakull, M. W., Soria, R., Grisé, F., \& Pietrzyński, G. 2014, Natur, 514, 198

Pintore, F., Belfiore, A., Novara, G., et al. 2018, MNRAS, 477, L90

Qiu, Y., Liu, J., Guo, J., \& Wang, J. 2015, ApJL, 809, L28

Rodríguez Castillo, G. A., Israel, G. L., Belfiore, A., et al. 2019, arXiv:1906. 04791

Sathyaprakash, R., Roberts, T. P., Walton, D. J., et al. 2019, MNRAS, 488, L35

Scargle, J. D. 1982, ApJ, 263, 835

Soderberg, A., Grindlay, J. E., Bloom, J. S., et al. 2009, astro2010: The Astronomy and Astrophysics Decadal Survey, Science White Papers No. 278

Sood, R., Farrell, S., O’Neill, P., \& Dieters, S. 2007, AdSpR, 40, 1528

Soria, R., Baldi, A., Risaliti, G., et al. 2007, MNRAS, 379, 1313

Soria, R., Kuntz, K. D., Winkler, P. F., et al. 2012, ApJ, 750, 152

Strickland, D. K., Colbert, E. J. M., Heckman, T. M., et al. 2001, ApJ, 560, 707

Tananbaum, H., Gursky, H., Kellogg, E. M., et al. 1972, ApJL, 174, L143

Tsygankov, S. S., Mushtukov, A. A., Suleimanov, V. F., \& Poutanen, J. 2016, MNRAS, 457, 1101

Urquhart, R., \& Soria, R. 2016, ApJ, 831, 56

van den Eijnden, J., Degenaar, N., Russell, T. D., et al. 2019, MNRAS, 483,4628

Vasilopoulos, G., Lander, S. K., Koliopanos, F., \& Bailyn, C. D. 2020, MNRAS, 491, 4949

Walton, D. J., Fürst, F., Bachetti, M., et al. 2016a, ApJL, 827, L13

Walton, D. J., Fürst, F., Harrison, F. A., et al. 2018, MNRAS, 473, 4360

Walton, D. J., Middleton, M. J., Pinto, C., et al. 2016b, ApJL, 826, L26

Walton, D. J., Middleton, M. J., Rana, V., et al. 2015, ApJ, 806, 65

Wilson-Hodge, C. A., Malacaria, C., Jenke, P. A., et al. 2018, ApJ, 863, 9 